\title{
IS A RIKITAKE DYNAMO IN SATURN'S INTERIOR AT THE ORIGIN OF THE VARIABILITY OF THE RADIO ROTATION PERIODS?
}

\author{
P. H. M. Galopeau*
}

\begin{abstract}
Recent observations performed by the radio and plasma wave science (RPWS) experiment on board the Cassini spacecraft have revealed the presence of two distinct and variable spin modulation periods (10.6 hours and 10.8 hours) in Saturn's radio emissions emanating from the northern and southern hemispheres respectively. The main time modulation of planetary radio emissions has always been attributed to the effect on the inner magnetosphere of the internal magnetic field which rigidly rotates with the deep interior of the planet. The magnetospheric plasma is supposed to be frozen in this magnetic field so that a north/south asymmetry in the radio modulation period should never be observed. However Saturn's magnetic field is very particular since its dipolar moment is nearly aligned with the rotation axis of the planet. Such an alignment could bring out some phenomena in the internal structure which are masked in the case of other magnetized planets the magnetic dipole of which is significantly tilted. The existence of two separated and slowly varying periods in the saturnian magnetic field could be the signature of a dynamo the dynamics of which is governed by a Rikitake system.
\end{abstract}

\section{Introduction}

The variability of Saturn's rotation period was detected for the very first time by Lecacheux et al. [1997] in observations of the Saturnian kilometric radiation (SKR) collected by the URAP experiment onboard the Ulysses spacecraft: a power spectrum analysis performed on a time period spreading from November 1, 1994 to January 31, 1997 revealed a radio period $1 \%$ longer than that derived from Voyager twenty years earlier. The same analysis performed on time series of $\sim 2$ months duration showed that this radio period was variable with time [Galopeau and Lecacheux, 2000]. In order to explain this variability of the observed period, the authors proposed an interpretation in terms of SKR

* LATMOS, Université Versailles Saint-Quentin-en-Yvelines, CNRS, 78280 Guyancourt, France 
source motion due to the solar wind variability, linked to a Kelvin-Helmholtz instability developing on the flank of the Saturnian magnetopause in the morning side. Under the influence of the solar wind fluctuations, the angular position in local time $\alpha$ of the radio source varies leading to a variable radio period $T_{\mathrm{SKR}}$ which can be linked to the sideral rotation period $T_{\text {sid }}$ of Saturn (supposed to be constant) by the formula: $T_{\mathrm{SKR}}=$ $T_{\text {sid }}\left(1+\dot{\alpha} T_{\text {sid }} / 2 \pi\right)$. Cecconi and Zarka [2005] studied in more details the systematic displacement of the radio source due to non-random variations of the solar wind velocity.

More recently, observations performed by the Cassini radio and plasma wave science (RPWS) experiment revealed the presence of two distinct and variable periods at 10.6 hours and 10.8 hours in Saturn's radio emissions produced in the northern and southern hemispheres respectively [Gurnett et al., 2009a, 2009b]. The main time modulation of planetary radio emissions is usually attributed to the effect of the internal magnetic field (rotating with the deep interior of the planet) on the inner magnetosphere. Such a north/south asymmetry in the radio rotation period should never be observed as the magnetospheric plasma is supposed to be frozen in the magnetic field.

In the following, we attempt to understand this phenomenon by involving the structure of the inner magnetic field of Saturn and the way it is generated.

\section{Particularity of Saturn's Magnetic Field}

Compared with the other magnetized planets in the solar system, a distinctive features of Saturn's inner magnetic field is that its dipole is nearly aligned with the rotation axis of the planet (see Figure 1). The measurements performed by the magnetometers embarked aboard Pioneer 11 and then aboard Voyager 1 and 2 revealed a tilt of the dipole axis smaller than 0.5 [Smith et al., 1980a, 1980b; Acuña and Ness, 1980; Acuña et al., 1981; Ness et al., 1981; Connerney et al., 1982]. This strong axisymmetry of the inner magnetic field was later confirmed by Cassini observations [Dougherty et al., 2005; Giampieri et al., 2006].

However, despite this special geometry of the magnetic field, the SKR presents a strong periodic time modulation which led some works to question the axial symmetry and to propose the existence of a magnetic field anomaly undetected by the spacecraft magnetometers [Galopeau et al., 1991; Galopeau and Zarka, 1992]. A model of Saturnian magnetic field including a magnetic anomaly (the strength of which decreases very quickly with the radial distance) was computed by these authors, based on a study of the SKR high frequency limit.

The periodic time modulation of the SKR as measured by Voyager led to the definition of Saturn's official rotation period (10 hr 39 min $22.4 \mathrm{~s}$ ). Recent works have shown that the SKR is not the only feature in Saturn's magnetosphere to present such a periodicity: indeed, the plasma and the magnetic field (and specially its azimuthal component $B_{\varphi}$ ) in the inner region of the Saturnian plasma disk are cyclic with the same variable period as the SKR [Gurnett et al., 2007]. Moreover Khurana et al. [2009] also showed that Saturn's middle magnetosphere displays periodicities close to the SKR period and semipermanent 


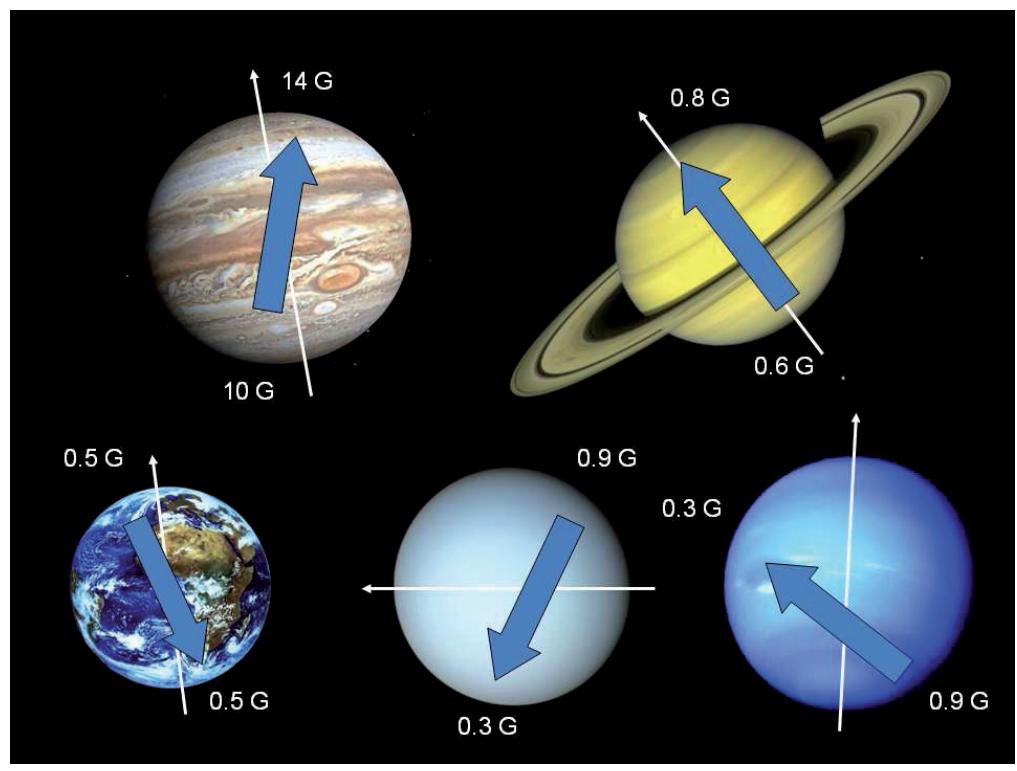

Figure 1: Magnetized planets of the solar system. The rotation axes, the magnetic dipoles and the maximum magnetic field intensities are indicated.

longitudinal asymmetries in the hot and energetic plasma population of the ring current region. The existence of this similar periodicity of the radio emissions, plasma, energetic particles and magnetic field suggests a single cause; but as the SKR is produced at the local gyrofrequency very near Saturn's body, the origin of the periodic time modulation can be attributed to the internal magnetic field generated in the deep interior of Saturn by a dynamo mechanism.

In this context, the presence in the SKR of two distinct variable periods has its origins directly in the variability of the planetary internal magnetic field and cannot be simply explained by a displacement in local time of the radio source as proposed before [Galopeau and Lecacheux, 2000; Cecconi and Zarka, 2005]: in such a scenario, the northern and southern sources would have correlated motions which would imply a single time period for both hemispheres.

\section{Dynamo Effect in Saturn's Interior}

When the magnetic dipole is significantly tilted relatively to the planetary rotation axis (e.g., the Earth and Jupiter), the entire magnetosphere is modulated at the spin rate of the planet. In the case of Saturn, the quasi-alignment of the magnetic dipole and the spin axis reveals more subtle effects, like a double periodicity linked to the generation of the internal field, which are usually masked. In the following, we introduce a basic model 
of dynamo which was applied in order to explain the polarity reversals of the terrestrial magnetic field.

\subsection{Dynamo General Equations in Axial Symmetry}

First we study the variation of the magnetic field $\mathbf{B}$ as a function of the velocity field $\mathbf{v}$. The basic MHD equations are respectively Faraday's law, Ohm's law and Ampère's law:

$$
\begin{aligned}
\frac{\partial \mathbf{B}}{\partial t} & =-\operatorname{curl} \mathbf{E} \\
\mathbf{j} & =\sigma(\mathbf{E}+\mathbf{v} \times \mathbf{B}) \\
\operatorname{curl} \mathbf{B} & =\mu_{0} \mathbf{j}
\end{aligned}
$$

leading to:

$$
\frac{\partial \mathbf{B}}{\partial t}=\operatorname{curl}(\mathbf{v} \times \mathbf{B})+\frac{1}{\mu_{0} \sigma} \Delta \mathbf{B} .
$$

To take into account the effect of the turbulence, we suppose that the velocity and the magnetic field are the sum of the average field and a fluctuation and we define the parameters $\alpha=\tau_{\text {corr }}\langle\delta \mathbf{v} \cdot \operatorname{curl} \delta \mathbf{v}\rangle, \eta_{T}=\tau_{\text {corr }}\langle\delta \mathbf{v} \cdot \delta \mathbf{v}\rangle$ and $\eta=\left(\mu_{0} \sigma\right)^{-1}+\eta_{T}$ so that equation (4) becomes:

$$
\frac{\partial \mathbf{B}}{\partial t}=\operatorname{curl}(\mathbf{v} \times \mathbf{B})+\operatorname{curl}(\alpha \mathbf{B})+\eta \Delta \mathbf{B} .
$$

The system is now supposed to present a direction of invariance (here the azimuthal direction), named toroidal direction; the perpendicular direction is named poloidal direction. We consider that the average fields $\mathbf{v}$ and $\mathbf{B}$ are the sum of a poloidal and a toroidal component: $\mathbf{v}=\mathbf{v}_{P}+\mathbf{v}_{T}, \mathbf{B}=\mathbf{B}_{P}+\mathbf{B}_{T}$ and $\mathbf{B}_{P}=$ curl $\mathbf{A}_{T}$. In the case of an axially symmetrical system, we introduce the following two scalar functions $B$ and $A$ fulfilling the conditions: $\mathbf{B}_{T}=B(r, z) \mathbf{e}_{\varphi}$ and $\mathbf{A}_{T}=A(r, z) \mathbf{e}_{\varphi}$, where $(r, \varphi, z)$ are the usual cylindrical coordinates.

By separating poloidal and toroidal components, equation (5) splits into two equations:

$$
\begin{aligned}
\frac{\partial \mathbf{B}_{P}}{\partial t} & =\operatorname{curl}\left(\mathbf{v}_{P} \times \mathbf{B}_{P}\right)+\operatorname{curl}\left(\alpha \mathbf{B}_{T}\right)+\eta \Delta \mathbf{B}_{P} \\
\frac{\partial \mathbf{B}_{T}}{\partial t} & =\operatorname{curl}\left(\mathbf{v}_{P} \times \mathbf{B}_{T}+\mathbf{v}_{T} \times \mathbf{B}_{P}\right)+\operatorname{curl}\left(\alpha \mathbf{B}_{P}\right)+\eta \Delta \mathbf{B}_{T} .
\end{aligned}
$$

The dynamo equations are direcly derived from (6) and (7):

$$
\begin{aligned}
\frac{\partial A}{\partial t}+\frac{1}{r}\left(\mathbf{v}_{P} \cdot \nabla\right)(r A) & =\alpha B+\eta \Delta^{*} A \\
\frac{\partial B}{\partial t}+r\left(\mathbf{v}_{P} \cdot \nabla\right)\left(\frac{B}{r}\right) & =r\left(\mathbf{B}_{P} \cdot \nabla\right)\left(\frac{v_{\varphi}}{r}\right)-\alpha \Delta^{*} A+\eta \Delta^{*} B
\end{aligned}
$$

where the operator $\Delta^{*}$ is defined by:

$$
\Delta^{*}=\frac{\partial^{2}}{\partial z^{2}}+\frac{\partial}{\partial r} \frac{1}{r} \frac{\partial}{\partial r} r .
$$




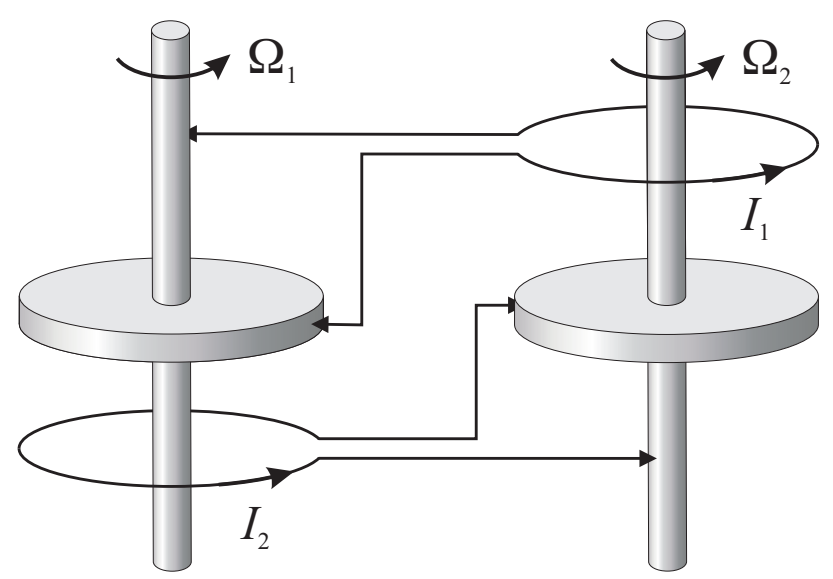

Figure 2: The Rikitake dynamo is composed of two disk dynamos coupled to one another.

\subsection{Rikitake Model}

Because equations (8) and (9) are very difficult to solve, early researchers searched for analogue systems which displayed the same temporal behavior. Following the study of a homopolar disk dynamo by Bullard [1955], Rikitake [1958] proposed a system consisting of two identical single Faraday-disk dynamos coupled together (see Figure 2).

The dynamos are driven by identical constant motor torques $G$ which maintain their motions. The equations governing the currents $I_{1}, I_{2}$ and the angular velocities $\Omega_{1}, \Omega_{2}$ are:

$$
\begin{array}{ll}
M \Omega_{1} I_{2}=L \frac{d I_{1}}{d t}+R I_{1} & C \frac{d \Omega_{1}}{d t}=G-M I_{1} I_{2} \\
M \Omega_{2} I_{1}=L \frac{d I_{2}}{d t}+R I_{2} & C \frac{d \Omega_{2}}{d t}=G-M I_{1} I_{2} .
\end{array}
$$

where $R$ and $L$ are respectively the resistance and the self inductance of each dynamo and $M$ the mutual inductance between them. $C$ is the moment of inertia.

This system can be simplified into the following dimensionless three equations:

$$
\begin{aligned}
& d x / d \tau=-\mu x+z y \\
& d y / d \tau=-\mu y+(z-a) x \\
& d z / d \tau=1-x y
\end{aligned}
$$

by introducing the new variables $x=(M / G)^{1 / 2} I_{1}, y=(M / G)^{1 / 2} I_{2}, z=(C M / G L)^{1 / 2} \Omega_{1}$ and $\tau=(G M / C L)^{1 / 2} t$, and the dimensionless constants $\mu=\left(C R^{2} / G L M\right)^{1 / 2}$ and $a=$ $(C M / G L)^{1 / 2}\left(\Omega_{1}-\Omega_{2}\right)$.

The solution is chaotic and tends to a strange attractor similar to the Lorenz one. An example of a numerical solution is displayed in Figure 3. The solution presents a pseudoperiodic behavior with a variable period; the polarity reverses after a random number of 

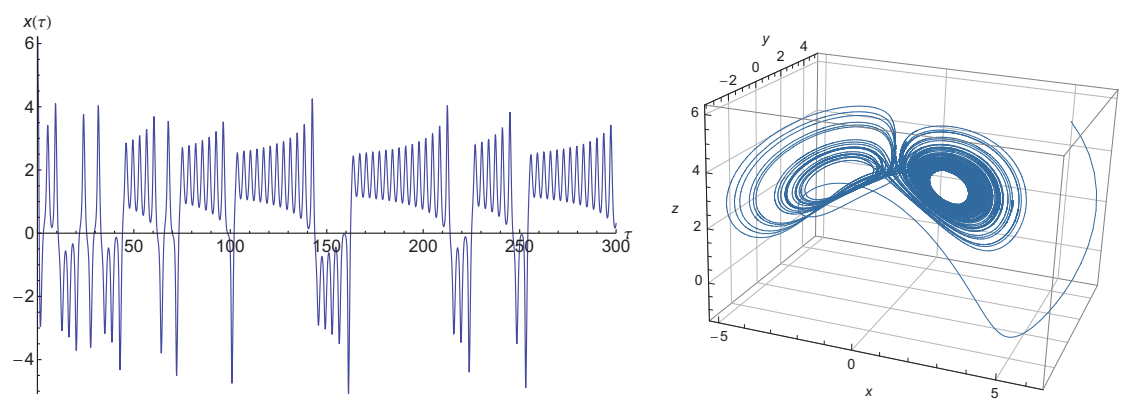

Figure 3: A typical solution of the Rikitake system obtained for $a=3, \mu=1$ and the initial conditions $x(0)=y(0)=z(0)=5$.

oscillations. The solution $[x(\tau), y(\tau), z(\tau)]$ 'coils itself' around two unstable stationary points [Cook and Roberts, 1970]. This simple model has been improved by introducing viscous friction and time delay [Cook, 1972] or even replaced by a multiple scale dynamo mechanism [Narteau et al., 2000].

\section{Results and Conclusion}

The solution of the Rikitake system consists in two variable currents $\left[I_{1}(t), I_{2}(t)\right]$ and two variable periods $\left[\Omega_{1}(t), \Omega_{2}(t)\right]$ which display a quasi-periodic behavior between the successive reversals. From equations (11) and (12), one can easily derive that $\Omega_{1}(t)-$ $\Omega_{2}(t)=a(G L / C M)^{1 / 2}$ is a constant. This particular property is quite reminiscent of the two variable periods observed in the SKR which practically satisfy $\Omega_{1}(t)+\Omega_{2}(t) \simeq$ constant. This last relation can be fulfilled by reversing one of the electric circuits of the Rikitake dynamo. In Figure 4, we display side by side the variation of the reduced rotation rate $z(\tau)$ of the Rikitake dynamo and the SKR variable periods published by Gurnett et al. [2009a]. By adjusting the time scale and the parameters of the dynamo, $\Omega_{1}(t)=(G L / C M)^{1 / 2} z(\tau)$ can fit the rotation rate of one of the SKR components (plotted in dashed line in Figure 4$)$. Then the other SKR period can be fitted by $\Omega_{2}(t)$.

The model presented in this paper shows that the time variation of the Saturnian internal magnetic field can explain the presence of the two distinct variable radio periods observed in the SKR. However the Rikitake model constitutes a first stage and is not good enough to fully describe Saturn's dynamo; a MHD approach taking into consideration the physical parameters of the interior of the planet (conductivity, viscosity, heat flux...) is required. The typical case of Saturn and its radio emissions shows us how it is important to study the interior structure for understanding some aspects of the magnetospheric physics. 


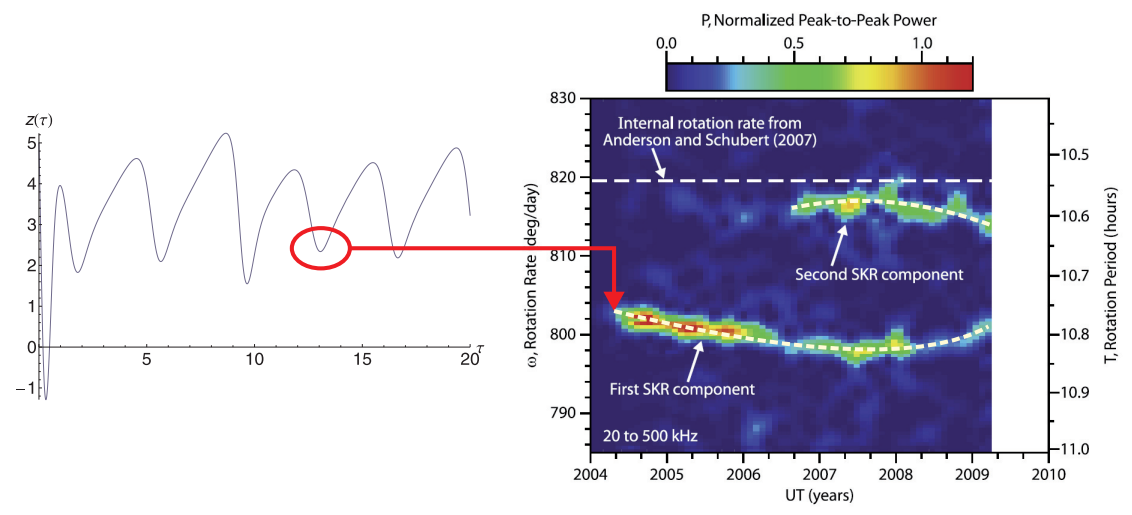

Figure 4: Comparison between the rotation rate $z(\tau)$ derived from the Rikitake system and that deduced from the southern component of SKR [Gurnett et al., 2009a].

\section{References}

Acuña, M.H., and N.F. Ness, The magnetic field of Saturn: Pioneer 11 observations, Science, 207, doi:10.1126/science.207.4429.444, 1980.

Acuña, M. H., J. E.P. Connerney, and N. F. Ness, Topology of Saturn's main magnetic field, Nature, 292, doi:10.1038/292721a0, 1981.

Bullard, E., The stability of a homopolar dynamo, Proc. Camb. Phil. Soc., 51, 04, doi:10.1017/S0305004100030814, 1955.

Cecconi, B., and P. Zarka, Model of a variable radio period for Saturn, J. Geophys. Res., 110, A9, A12203, doi:10.1029/2005JA011085, 2005.

Connerney, J.E.P., N.F. Ness, and M.H. Acuña, Zonal harmonic model of Saturn's magnetic field from Voyager 1 and 2 observations, Nature, 298, 44-46, doi:10.1038/ $298044 \mathrm{a} 0,1982$.

Cook, A. E., Two disc dynamos with viscous friction and time delay, Proc. Camb. Phil. Soc., 71, 01, doi:10.1017/S0305004100050374, 1972.

Cook, A. E., and P. H. Roberts, The Rikitake two-disc dynamo system, Proc. Camb. Phil. Soc., 68, 02, doi:10.1017/S0305004100046338, 1970.

Dougherty, M. K., et al. (17 co-authors), Cassini magnetometer observations during Saturn orbit insertion, Science, 307, doi:10.1126/science.1106098, 2005.

Galopeau, P., and P. Zarka, Reply to comment on 'Evidence of Saturn's magnetic field anomaly from Saturnian kilometric radiation high-frequency limit', J. Geophys. Res., 97, 12, doi:10.1029/92JA00323, 1992. 
Galopeau, P. H. M., and A. Lecacheux, Variations of Saturn's radio rotation period measured at kilometer wavelengths, J. Geophys. Res., 105, 13, 089-13, 102, doi: 10.1029/1999JA005089, 2000.

Galopeau, P., P. Zarka, and A. Ortega-Molina, Evidence of Saturn's magnetic field anomaly from saturnian kilometric radiation high-frequency limit, J. Geophys. Res., 96, 14, 129-14, 140, 1991.

Giampieri, G., M.K. Dougherty, E.J. Smith, and C.T. Russell, A regular period for Saturn's magnetic field that may track its internal rotation, Nature, 441, doi: 10.1038/nature04750, 2006.

Gurnett, D. A., A.M. Persoon, W.S. Kurth, J. B. Groene, T. F. Averkamp, M. K. Dougherty, and D. J. Southwood, The variable rotation period of the inner region of Saturn's plasma disk, Science, 316, doi:10.1126/science.1138562, 2007.

Gurnett, D. A., A. Lecacheux, W. S. Kurth, A. M. Persoon, J. B. Groene, L. Lamy, P. Zarka, and J. F. Carbary, Discovery of a north-south asymmetry in Saturn's radio rotation period, Geophys. Res. Lett., 36, L16102, doi:10.1029/2009GL039621, 2009a.

Gurnett, D. A., A. M. Persoon, J. B. Groene, A. J. Kopf, G. B. Hospodarsky, and W. S. Kurth, A north-south difference in the rotation rate of auroral hiss at Saturn: Comparison to Saturn's kilometric radio emission, Geophys. Res. Lett., 36, L21108, doi: 10.1029/2009GL040774, 2009b.

Khurana, K. K., D. G. Mitchell, C. S. Arridge, M. K. Dougherty, C. T. Russell, C. Paranicas, N. Krupp, and A. J. Coates, Sources of rotational signals in Saturn's magnetosphere, J. Geophys. Res., 114, A13, A02211, doi:10.1029/2008JA013312, 2009.

Lecacheux, A., P. H. M. Galopeau, and M. Aubier, Re-visiting Saturnian kilometric radiation with Ulysses/URAP, in Planetary Radio Emissions IV, edited by H. O. Rucker, S. J. Bauer, and A. Lecacheux, 313-325, Austrian Academy of Sciences Press, Vienna, 1997.

Narteau, C., E. Blanter, J.-L. Le Mouël, M. Shirnman, and C. J. Allègre, Reversal sequence in a multiple scale dynamo mechanism, Phys. Earth Planet. Inter., 120, 4, doi:10.1016/S0031-9201(00)00145-X, 2000.

Ness, N. F., M. H. Acuña, R. P. Lepping, J. E. P. Connerney, K. W. Behannon, L. F. Burlaga, and F.M. Neubauer, Magnetic field studies by Voyager 1: Preliminary results at Saturn, Science, 212, doi:10.1126/science.212.4491.211, 1981.

Rikitake, T., Oscillations of a system of disk dynamos, Proc. Camb. Phil. Soc., 54, 01, doi:10.1017/S0305004100033223, 1958.

Smith, E. J., L. Davis, D. E. Jones, P. J. Coleman, D. S. Colburn, P. Dyal, and C. P. Sonett, Saturn's magnetic field and magnetosphere, Science, 207, 407-410, doi:10.1126/ science.207.4429.407, 1980a.

Smith, E. J., L. Davis, D. E. Jones, P. J. Coleman, D. S. Colburn, P. Dyal, and C. P. Sonett, Saturn's magnetosphere and its interaction with the solar wind, J. Geophys. Res., 85, doi:10.1029/JA085iA11p05655, 1980b. 